\title{
Multitarget-directed ligands for neurodegenerative diseases: real opportunity or blurry mirage?
}

\author{
Mohamed Benchekroun*,1 \& Samuele Maramai**,2 \\ ${ }^{1}$ Institut de Chimie des Substances Naturelles, CNRS UPR 2301, Université Paris-Saclay, Avenue de la Terrasse, Gif-sur-Yvette, \\ 91198, France \\ ${ }^{2}$ Sussex Drug Discovery Centre, School of Life Sciences, University of Sussex, Brighton, BN1 9QJ, UK \\ *Author for correspondence: Tel.: +33 169824 585; mohamed.benchekroun@cnrs.fr \\ **Author for correspondence: Tel.: +44 127387 7386; s.maramai@sussex.ac.uk
}

\begin{abstract}
“ an alternative to the mono-target approach could take into consideration the multifactorial character of neurodegenerative diseases by developing molecules, which have different kinds of pharmacophores, and are able to interact with different biological targets or events”
\end{abstract}

First draft submitted: 14 June 2018; Accepted for publication: 7 December 2018; Published online: 14 February 2019

Keywords: $\beta$-amyloid $\bullet$ Alzheimer's $\bullet$ drug discovery $\bullet$ inhibitor $\bullet$ metal dishomeostasis $\bullet$ mitochondria $\bullet$ multitargetdirected ligands $\bullet$ neurodegenerative diseases $\bullet$ neuronal signaling $\bullet$ oxidative stress

\section{Neurodegenerative diseases: a long road ahead to find new medicines}

Neurodegenerative diseases such as Alzheimer's, Parkinson's and Huntington's diseases and multiple sclerosis (AD, PD, HD and MS, respectively) currently represent a heavy economic and social burden for our societies. The number of people suffering from AD, for example, is expected to reach 82 million in 2030 and 152 million in 2050. Medical treatment and care costs were estimated to exceed 800 billion US dollars in 2015, and are predicted to attain the stratospheric amount of 2 trillion US dollars by 2030; all of this without forgetting the great distress experienced by most families and carers [1]. Taking into account the global increase in lifespan worldwide, tremendous efforts must be made to accompany and treat the affected patients.

In this regard, medicinal chemists play a central role in delivering new molecules capable of treating and/or curing neurodegenerative diseases by targeting recognized and common pathological features, such as protein misfolding, metal-mediated free radical production, metal dishomeostasis and mitochondrial dysfunction. However, there is still work to be done to fully unveil a more precise etiologic cascade of these pathologies. AD for instance possesses a highly intricate and complex etiology where various biological and histological phenomena, such as $A \beta$ plaques deposits, cholinergic deficit and oxidative stress, are intimately interconnected at various levels.

Until now, the US FDA- and European Medicines Agency (EMA)-approved drugs that used to treat some neurodegenerative diseases are targeting one biological event at a time, leading to therapies that, in most cases, only attenuate symptoms and fairly ameliorate day-to-day life. Good examples are donepezil and rivastigmine, two potent $\mathrm{AChE}$ inhibitors for $\mathrm{AD}$ treatment. In addition, the pharmaceutical industry is now facing a significant rate of attrition in $\mathrm{AD}$ drug discovery, with several drugs failing in the clinics. Examples of this include small molecules inhibitors of $\beta$-secretase (such as verubecestat [2]), modulators of $\gamma$-secretase (such as avagacestat [3]) and the highly sought after anti-A $\beta$ immunotherapeutics (such as solanezumab [4]). The underlying conclusion is that a profound reshape of the way the problem is tackled is necessary to avoid further failures, including a better understanding of the onset and progression of the disease.

\section{Genesis \& development of multitarget-directed ligands}

From a medicinal chemistry perspective, an alternative to the mono-target approach could take into consideration the multifactorial character of neurodegenerative diseases by developing molecules, which have different kinds of pharmacophores, and are able to interact with different biological targets or events. In 2008, Melchiorre and 
colleagues termed these as the multitarget-directed ligands (MTDLs) [5]. Since then, many new MTDLs which hit various targets, such as cholinesterase enzymes, $\mathrm{A} \beta$ peptides and hyperphosphorylated $\tau$ protein, $\mathrm{Ca}^{2+}$ channel, endogenic amines receptors (for example, serotonine, dopamine or acetylcholine), endogenic metals $\left(\mathrm{Cu}^{2+} \mathrm{or} \mathrm{Fe}^{2+}\right.$ ) as well as the related downstream processes, such as neuroinflammation and oxidative stress have been described [6,7].

In an ideal world, the compound would therefore be able to modulate all of these biological processes while also possessing adequate in vitro or in vivo metabolic and toxicology profiles. However, the reality is quite different and as pointed out recently by Oset-Gasque and Marco-Contelles [8], this is simply not feasible. The true challenge in this field lies in identifying the judicious combination of targets or events to modulate in a synergistic way that can ultimately yield a drug candidate.

In this light, Benchekroun $e t$ al. have recently disclosed the antioxidant additive approach for AD treatment by developing cholinesterase inhibitors acting as direct antioxidants by scavenging reactive oxygen species and activating the nuclear factor $\mathrm{Nrf2}$ transcriptional pathway responsible of the production of endogenous antioxidant enzymes (for example, HO-1, GST, NQO-1). Thus, by targeting different facets of oxidative stress, these MTDLs represent interesting prototypes for the development of clinical candidates which have antioxidant, neuroprotective effects and can penetrate effectively the CNS and restore cognition and memory functions [9].

Following a similar way, several bis-tacrine-based compounds bearing a specifically designed peptidic motif able to inhibit the catalytic and noncatalytic functions of human AChE have been reported. Worth of note, the binding of these multifunctional tools with peripheral sites of human $\mathrm{AChE}$ leads to specific interference with $\mathrm{A} \beta$ aggregation and self-oligomerization processes. In fact, by physically hampering $A \beta$ binding to the enzyme surface, the peptidic backbone of these compounds is likely to be responsible for the observed reduction of AChE-induced $A \beta$ aggregation. Moreover, these analogs are also capable of interfering with $A \beta$ oligomerization, thus reducing oligomers-mediated toxicity, and disrupting pre-formed $A \beta$ fibrils $[10,11]$.

MTDLs may also have a great impact on other neurodegenerative diseases. Interestingly, some very recent studies proposed dual inhibitors of A2AA receptors and MAO-B as useful tools against PD [12]. According to the authors, a dual A2AA/MAO-B inhibitor would have the neuroprotective effects of A2AAR antagonism combined with the advantages of a maintained dopaminergic tone due to MAO-B inhibition. Even if these lead compounds need further optimization, they represent a good starting point for the development of multitarget compounds endowed with anti-PD activity.

Finally, a very promising example of MTDL is contilisant, a multipotent molecule inhibiting the cholinesterases and MAOs enzymes, as well as showing high affinity at H3R and selective S1R agonism [13]. This drug is not only endowed with antioxidant and neuroprotective effects, but is also able to alleviate the cognitive deficit induced by $A \beta_{1-42}$ in a mouse model of dementia, thus indicating its potential use in the development of drugs against $A D$.

\section{Conclusion}

So far, the literature is full of examples regarding MTDLs as potential and effective treatments for neurodegenerative diseases. However, to what extend can a compound be multipotent? How can we make a compound selective enough to not interact with other systems that are irrelevant for the disease or important to sustain crucial biological processes? What off-target liabilities are acceptable throughout the discovery of a nootropic and neuroprotectant drug? These questions need to be raised, as they represent serious hurdles during the development of the drug candidates. Moreover, setting up too many pharmacophores into a single molecule could further augment the odds of expressing undesired off-target liabilities, translating into a more difficult pharmacological and drug metabolism-pharmacokinetics assessment - which may possibly lead to undesired side effects in the clinics.

This might, in part, discourage the pharmaceutical industry and CROs to follow the path for the development of multitarget compounds. However, the concept of selective drugs in neurodegeneration has drastically changed in the last decade. The multifactorial complexity of neurodegenerative diseases has altered the common presupposition that 'dirty drugs' cannot represent useful tools in therapy [14].

Therefore, beyond being simply a catch-all strategy only studied in some academic settings, we strongly believe that the multitarget approach will soon yield new therapies against neurodegenerative diseases able to halt or reverse cognitive impairment, ataxia and restore the brain function. The careful identification of the key events or biological targets to mitigate as well as the estimation of the efficacy and safety parameters throughout the preclinical and clinical phases is of the utmost importance. This can only be done with a constant and supportive collaboration between medicinal chemists, pharmacologists, biochemists, biologists and medical practitioners. Then maybe, the blurry mirage can be translated into a tangible reality. 


\section{Financial \& competing interests disclosure}

The authors have no relevant affiliations or financial involvement with any organization or entity with a financial interest in or financial conflict with the subject matter or materials discussed in the manuscript. This includes employment, consultancies, honoraria, stock ownership or options, expert testimony, grants or patents received or pending, or royalties.

No writing assistance was utilized in the production of this manuscript.

\section{References}

1. WHO. Dementia. www.who.int/mediacentre/factsheets/fs362/en/

2. Egan MF, Kost J, Tariot PN et al. Randomized trial of verubecestat for mild-to-moderate Alzheimer's disease. N. Engl. J. Med. 378(18), 1691-1703 (2018).

3. Coric V, Salloway S, van Dyck CH et al. Targeting prodromal Alzheimer disease with avagacestat: a randomized clinical trial. JAMA Neurol. 72(11), 1324-1333 (2015).

4. Honig LS, Vellas B, Woodward M et al. Trial of Solanezumab for mild dementia due to Alzheimer's disease. N. Engl. J. Med. 378(4), 321-330 (2018).

5. Cavalli A, Bolognesi ML, Minarini A et al. Multi-target-directed ligands to combat neurodegenerative diseases. J. Med. Chem. 51(3), 347-372 (2008).

6. León R, Garcia AG, Marco-Contelles J. Recent advances in the multitarget-directed ligands approach for the treatment of Alzheimer's disease. Med. Res. Rev. 33(1), 139-189 (2013).

7. Ismaili L, Refouvelet B, Benchekroun $\mathrm{M}$ et al. Multitarget compounds bearing tacrine- and donepezil-like structural and functional motifs for the potential treatment of Alzheimer's disease. Prog. Neurobiol. 151, 4-34 (2017).

8. Oset-Gasque MJ, Marco-Contelles J. Alzheimer's disease, the "one-molecule, one-target" paradigm, and the multitarget directed ligand approach. ACS Chem. Neurosci. 9(3), 401-403 (2018).

9. Benchekroun M, Romero A, Egea J et al. The antioxidant additive approach for Alzheimer's disease therapy: new ferulic (lipoic) acid plus melatonin modified tacrines as cholinesterases inhibitors, direct antioxidants, and nuclear factor (erythroid-derived 2)-like 2 activators. $J$. Med. Chem. 59(21), 9967-9973 (2016).

10. Butini S, Brindisi M, Brogi S et al. Multifunctional cholinesterase and amyloid beta fibrillization modulators. Synthesis and biological investigation. ACS Med. Chem. Lett. 4(12), 1178-1182 (2013).

11. Brogi S, Butini S, Maramai S et al. Disease-modifying anti-Alzheimer's drugs: inhibitors of human cholinesterases interfering with $\beta$-amyloid aggregation. CNS Neurosci. Ther. 20(7), 624-632 (2014).

12. Jaiteh M, Zeifman A, Saarinen M et al. Docking screens for dual inhibitors of disparate drug targets for Parkinson's disease. J. Med. Chem. 61(12), 5269-5278 (2018).

13. Bautista-Aguilera M Ó, Hagenow S, Palomino-Antolin A et al. Multitarget-directed ligands combining cholinesterase and monoamine oxidase inhibition with histamine H3R antagonism for neurodegenerative diseases. Angew. Chem. Int. Ed. Engl. 56(41), 12765-12769 (2017).

14. Ramsay RR, Majekova M, Medina M, Valoti M. Key targets for multi-target ligands designed to combat neurodegeneration. Front. Neurosci. 10, doi:10.3389/fnins.2016.00375 (2016) (Online article) 
